\title{
Protocol
}

\section{Optimized Affinity Capture of Yeast Protein Complexes}

\author{
John LaCava, Javier Fernandez-Martinez, Zhanna Hakhverdyan, and Michael P. Rout ${ }^{1}$ \\ Laboratory of Cellular and Structural Biology, The Rockefeller University, New York, New York 10065
}

Here, we describe an affinity isolation protocol. It uses cryomilled yeast cell powder for producing cell extracts and antibody-conjugated paramagnetic beads for affinity capture. Guidelines for determining the optimal extraction solvent composition are provided. Captured proteins are eluted in a denaturing solvent (sodium dodecyl sulfate polyacrylamide gel electrophoresis sample buffer) for gel-based proteomic analyses. Although the procedures can be modified to use other sources of cell extract and other forms of affinity media, to date we have consistently obtained the best results with the method presented.

\section{MATERIALS}

It is essential that you consult the appropriate Material Safety Data Sheets and your institution's Environmental Health and Safety Office for proper handling of equipment and hazardous material used in this protocol.

Reagents

Antibody-conjugated paramagnetic beads

There are many choices in paramagnetic media available commercially. We prefer Life Technologies Dynabeads M-270 Epoxy Beads for conjugating to our own choice of antibody in-house. We suggest using $300 \mathrm{mg}$ of antibody-conjugated beads combined with $2 \mathrm{~mL}$ of storage buffer or equivalent slurry (i.e., a 15\% w:V suspension). For the conjugation procedure, the manufacturer's instructions may be followed with excellent results or see Cristea and Chait (2011).

Extraction solvent (e.g., one of the following):

- $40 \mathrm{~mm}$ Tris-Cl (pH 8.0) or $40 \mathrm{~mm}$ Na-HEPES (pH 7.4), 0.15-1 M NaCl, and 0.1\% (v/v) Tween 20

- $0.25-1.5 \mathrm{M}$ ammonium acetate (pH close to 7.0) and 1\% (v/v) Triton X-100

- $250 \mathrm{~mm}$ sodium phosphate (pH 7-8) and 1\% (v/v) Triton X-100

- 40 mм Tris- $\mathrm{Cl}$ ( $\mathrm{pH} 8.0$ ), 50-250 mm sodium citrate, $150 \mathrm{~mm}$ sodium chloride, and 1\% (v/v) Triton X-100

Determine the optimal extraction solvent composition for each individual protein complex of interest. Use empirical and practical considerations, including prior art gleaned from the literature, to select an appropriate composition. Above we suggest four kinds of formulation, with the ranges of concentrations to be explored for each. They have proven very useful in our hands for a wide variety of yeast complexes. These formulations are reasonable starting points, but they are not universal and may need modifications (e.g., using a different detergent, buffer agent, or pH). For additional discussion on this topic, see Introduction:

\footnotetext{
${ }^{1}$ Correspondence: rout@rockefeller.edu

(C) 2016 Cold Spring Harbor Laboratory Press

Cite this protocol as Cold Spring Harb Protoc; doi:10.1101/pdb.prot087932
} 
J. LaCava et al.

Protein Complex Purification by Affinity Capture [LaCava et al. 2015a]). Use the extraction solvent at room temperature.

Mass-spectrometry-compatible protein stain (for staining proteins in sodium dodecyl sulfate polyacrylamide gel electrophoresis [SDS-PAGE] gels)

We recommend the Blue Silver modified Neuhoff's colloidal Coomassie Blue G-250 stain (Candiano et al. 2004) or Imperial Protein Stain (Thermo Scientific 24615).

Protease inhibitor cocktail

We recommend using an EDTA-free cocktail unless EDTA is specifically required in the optimized extraction buffer. We commonly use product $P 8340$ from Sigma-Aldrich or cOmplete mini, EDTA-free protease inhibitor cocktail tablets from Roche. The protease inhibitors should be reconstituted directly in extraction solvent at the volume recommended by the manufacturer, or in the case of Roche tablets, prepared as a 100x concentrate in water or the extraction solvent and diluted to $1 \times$ with the same solvent before use. $100 \times$ stocks prepared in water are more versatile to use with multiple different solvents and only impart a 1\% dilution on other ingredients.

SDS-PAGE sample buffer $(1.1 \times)$

We use $4 X$ NUPAGE LDS Sample Buffer (ThermoFisher Scientific NP0008) and dilute it to $1.1 \times$ just before use. The $0.1 \times$ "extra" is to account for the reducing agent that is added in Step 12.

SDS-PAGE system

Any SDS-PAGE system can be used for the final separation of protein complex constituents. However, for our routine separations, we prefer the Novex NuPAGE system (Life Technologies) with 1-mm-thick, 4\%-12\% BisTris gels run in 2-( $\mathrm{N}$-morpholino)ethanesulfonic acid- or 3-(N-morpholino)propanesulfonic acid-SDS buffer. Final elution is achieved by adding SDS (or lithium dodecyl sulfate) containing gel-loading buffer.

Yeast strain of interest

Equipment

Analytical balance (with milligram accuracy)

Heat block (set at $70^{\circ} \mathrm{C}$ )

Liquid nitrogen

Microcentrifuge (14,000 rpm) (e.g., 5417R from Eppendorf with F-45-30-11 rotor)

Microcentrifuge tubes (1.5- or 2-mL; screw cap recommended)

Micropipettors and tips (e.g., P1000, P200, and P20)

Neodymium magnet (e.g., DynaMag-2 from Life Technologies)

Planetary ball mill (PM100 from Retsch)

Many mechanical milling devices are available, but we use a Retsch PM100 planetary ball mill to produce a micron-scale powder with excellent properties for subsequent affinity capture, providing high-yield, low-noise affinity capture results.

Rotating wheel mixer (or shaker) (set at $4^{\circ} \mathrm{C}$ )

SDS-PAGE equipment (for one-dimensional gels)

Spatula (small)

Volumetric spoon (e.g., Norpro 3080 Mini Measuring Spoons from Amazon)

Vortex mixer

METHOD

\section{Cryogenic Disruption of Yeast Cells}

1. Grow a yeast cell culture to a density of at least $3.0 \times 10^{7}$ cells $/ \mathrm{mL}$. Prepare a washed cell pellet that is free of excess liquid and resembles a thick paste (e.g., by centrifugation at $\sim 3000 \mathrm{~g}$ for $5 \mathrm{~min}$ at $4^{\circ} \mathrm{C}$ ). Press the cell paste through an appropriately sized (e.g., 10-, 20-, or 50-mL) syringe into liquid nitrogen in a clean Dewar flask to generate frozen yeast cell "noodles."

The precise cell culture density can be changed to suit your biological inquiry. We suggest processing a total yeast pellet of a minimum of $\sim 5 \mathrm{~g}$ wet cell weight, up to tens of grams, total yeast pellet be processed at a time. 
2. Prechill all components of the planetary ball mill in liquid nitrogen in a polystyrene container. Add frozen noodles to the milling jar $(\leq 20 \mathrm{~mL}$ of noodles/50-mL jar and $20-50 \mathrm{~mL}$ of noodles/ $125-\mathrm{mL}$ jar). Weigh the milling jar with noodles and adjust the counterbalance weight. Add seven to eleven $20-\mathrm{mm}$ stainless steel balls to the $125-\mathrm{mL}$ jar (depending on the volume of noodles) or three balls to the $50-\mathrm{mL}$ jar.

Ensure no liquid nitrogen is visible within the jar before milling. This is important to avoid venting of cell material during milling or opening of the jar. The milling jar for the PM100 is not airtight, and under high pressure, nitrogen gas will escape. However, although it is unlikely, excess pressure buildup could lead to an explosion. Therefore, if liquid nitrogen enters the jar and is visible, be cautious, and allow it to evaporate before initiating milling.

3. Perform milling in eight cycles. For the $125-\mathrm{mL}$ jar, use the following settings for each cycle: $400 \mathrm{rpm}, 3 \mathrm{~min}$, and $1 \mathrm{~min}$ reverse rotation with no breaks between rotations. For the $50-\mathrm{mL}$ jar, use the following: $500 \mathrm{rpm}, 3 \mathrm{~min}$, and $1 \mathrm{~min}$ reverse rotation with no breaks between rotations. Cool the jars in liquid nitrogen between cycles. Do not remove the lid because this may result in cell loss. Keep the lid chilled by carefully pouring liquid nitrogen over the top of the jar. Do not submerge the jar completely because liquid nitrogen may leak into the milling chamber.

YOu MUST hear the balls rattling around in the jar. If there is no rattling, add/remove balls until you hear a rattle. It is not considered a milling cycle unless there is rattling.

4. When eight cycles are complete, remove the cryomilled yeast cell powder from the jars with a spatuala. Weigh the powder into microcentrifuge tubes using a spatula or volumetric spoon. Use liquid nitrogen to prechill all utensils and tubes, and return the utensils to liquid nitrogen in between uses. Tare an analytical balance with each prechilled tube before adding the cell powder, and then add the cell powder and check the mass added by quickly replacing the tube with powder on the balanced scale. Work quickly to avoid thawing the cell powder. Keep cell powder stocks and aliquots transferred to microcentrifuge tubes in liquid nitrogen.

Using the measuring spoons suggested in the Equipment list, we have found that one heaped scoop using the spoon labeled "smidgen" gives 50 mg of yeast cell powder and one level scoop using the spoon labeled "dash" gives 250 mg of yeast cell powder. However, an initial feel for the "size" of the scoop must be made by the user. We have found that $100 \mathrm{mg}$ of cryomilled yeast cell powder is commonly sufficient to purify a tagged protein complex of a moderate to low abundance at levels detectable by Coomassie blue staining after SDS-PAGE. Depending on the abundance of the complex and the application, the amount of starting material may need to be increased. If $>250 \mathrm{mg}$ needs to be used, we recommend weighing out multiple 250-mg samples and pooling the eluates at the end.

Store the cryomilled yeast cell powder for up to $1 \mathrm{yr}$ at $-80^{\circ} \mathrm{C}$ or proceed immediately to Step 5.

\section{Preparation of Yeast Cell Extracts}

5. Remove the tubes containing aliquots of yeast cell powder from liquid nitrogen and let them stand with open (or loosened) caps for $\sim 30 \mathrm{sec}$ (per $100 \mathrm{mg}$ ) at room temperature.

Residual pressure due to evaporating liquid nitrogen held within the cell powder can cause standard microcentrifuge tubes to pop open during warming, potentially resulting in the loss of the cell powder. Snap-cap tubes can become brittle at the temperature of liquid nitrogen, causing the hinge to break. This is why screw caps are preferred at this step. No thawing is observed during this brief incubation at room temperature, but be mindful not to initiate thawing of more material than you can process rapidly in Step 6.

6. Add four volumes of extraction solvent at room temperature supplemented with protease inhibitors to the yeast cell powder (e.g., $400 \mu \mathrm{L}$ per $100 \mathrm{mg}$ of powder). Briefly vortex $(\sim 30 \mathrm{sec})$ to completely resuspend the cell powder.

It is important to use extraction solvent at room temperature to expedite the resuspension and avoid ice formation at the powder/liquid interface. Keep resuspended crude extracts on ice until all samples are ready (but try to process all as rapidly as possible). Following this step, the extraction solvent can be held on ice to be used for subsequent washes.

7. Clarify the crude extracts by spinning in a benchtop microcentrifuge at $14,000 \mathrm{rpm}$ for $7 \mathrm{~min}$ at $4^{\circ} \mathrm{C}$. 
J. LaCava et al.

Centrifuging in an Eppendorf 5417R with an F-45-30-11 rotor (or similar microcentrifuge) under these conditions is roughly equivalent to centrifuging in a Beckman JA-25.50 rotor at 40,000g for 15 min.

Perform Steps 8 and 9 while the extracts are centrifuging. Proceed immediately to affinity capture.

\section{Equilibration of Antibody-Conjugated Beads}

8. While the cell extracts are in the centrifuge, aliquot the magnetic affinity medium into microcentrifuge tubes.

Typically $5 \mu \mathrm{L}$ of antibody-conjugated magnetic beads (suspended in a 15\% w:V slurry) per $100 \mathrm{mg}$ of frozen powder provides an excellent depletion ( $\geq 70 \%)$ of the target protein and its accompanying complex(es) from the clarified extract. However, a titration of the quantity of affinity medium needed to efficiently deplete the tagged protein can be performed and monitored by western blot. As the quantity of beads increases relative to extract, the opportunity for off-target protein binding (i.e., experimental noise) increases, potentially polluting the fraction. Thus, a compromise between complete recovery and increasing noise is usually sought; we recommend operating at 70\%-95\% depletion efficiency as often as possible. You may also want to assess the initial degree of extraction of the tagged protein (i.e., what fraction remains in the pellet versus the supernatant), keeping in mind that greater extraction of the tagged protein into the solvent does not necessarily translate to greater stability of the complex(es) it forms.

9. Pre-equilibrate the beads by washing them three times with $0.5 \mathrm{~mL}$ of extraction solvent. To wash the beads, agitate by vortexing to fully resuspend, then put the tube on the magnet briefly to allow the beads to collect on the tube wall, and remove the supernatant. To avoid excessive drying of the affinity medium, remove the final wash as the centrifugation in Step 7 reaches completion.

In Step 9 as in Step 11, agitating the beads excessively can cause some of the solution to collect away from the test in the case or on the side of the tube. The user should always ensure all the solution is at the bottom of the tube, typically by brief pulse centrifugation in a low-speed bench-top minicentrifuge.

10. Without disturbing the pellets, transfer the clarified extracts from Step 7 to the tubes containing the washed and equilibrated affinity medium from Step 9. Save an aliquot from each sample before combining with the affinity medium for analysis by western blot, if necessary. Incubate the mixture on a rotating wheel mixer for $30 \mathrm{~min}$ at $4^{\circ} \mathrm{C}$ (batch binding).

Although tagged protein binding may increase over several hours, the accumulation of off-target binding often increases disproportionately (Cristea et al. 2005). We advise using the shortest incubation time that gives an excellent depletion with low off-target binding-typically between $15 \mathrm{~min}$ and $1 \mathrm{~h}$.

11. After the batch binding is complete, place the tubes on a magnet to collect the beads and remove the supernatant (save an aliquot for analysis by western blot, if necessary). Wash the beads three times with $500 \mu \mathrm{L}$ of extraction buffer. (This is best accomplished by pipetting the beads up and down or by brief, low-speed vortex mixing. High-speed vortex mixing is not recommended at this stage because, in our experience, some components may be released from the immobilized complexes, presumably because of shearing forces.) During the second wash, transfer the beads to a fresh microcentrifuge tube, and then perform the final wash.

Changing to a fresh tube helps to reduce the background due to nonspecific adsorption of proteins to the tube walls. The wash volume may be increased up to the nominal volume of the tube for effectively washing larger quantities of affinity medium.

At this point, the sample can be eluted using denaturing conditions (see Step 12) or using nondenaturing (native) conditions; for the latter, see Protocol: Native Elution of Yeast Protein Complexes Obtained by Affinity Capture (LaCava et al. 2015b).

\section{Elution (Denaturing Conditions)}

12. Elute the protein complexes by incubating the beads with $20 \mu \mathrm{L}$ of $1.1 \times$ SDS-PAGE sample buffer (without a reducing agent to avoid excessive release of Ig chains into the eluted fraction; 1/10th volume of reducing agent will be added later). Scale the volume of sample buffer according to the quantity of beads and the size of the gel well. (The volume should be sufficient to resuspend the beads and large compared to the residual volume retained by the beads.) To release complexes captured via a protein A tag from magnetic media conjugated to rabbit IgG, incubate for $10 \mathrm{~min}$ 
at room temperature. To facilitate the release of complexes captured via a GFP tag, depending on the affinity of the anti-GFP antibody, it may be necessary to heat at $70^{\circ} \mathrm{C}$. Agitate gently during elution. Place the tubes on the magnet and recover the eluted fraction. Incubate the eluates with a reducing agent (typically, heat for $10 \mathrm{~min}$ at $70^{\circ} \mathrm{C}$ ) and perform SDS-PAGE and staining to assess the composition of the purified complex.

\section{RELATED INFORMATION}

For further information regarding this procedure, consult Oeffinger et al. (2007); Domanski et al. (2012), and LaCava et al. (2013). The latest versions of our detailed protocols for cryomilling yeast and conjugating antibodies to Dynabeads can be found on our website (http://www.ncdir.org). These protocols evolve with our work and are continuously updated online as improvements are achieved.

\section{REFERENCES}

Candiano G, Bruschi M, Musante L, Santucci L, Ghiggeri GM, Carnemolla B, Orecchia P, Zardi L, Righetti PG. 2004. Blue silver: A very sensitive colloidal Coomassie G-250 staining for proteome analysis. Electrophoresis 25: 1327-1333.

Cristea IM, Chait BT. 2011. Conjugation of magnetic beads for immunopurification of protein complexes. Cold Spring Harb Protoc doi: $10.1101 /$ pdb.prot5610.

Cristea I, Williams R, Chait B, Rout M. 2005. Fluorescent proteins as proteomic probes. Mol Cell Proteomics 4: 1933-1941.

Domanski M, Molloy K, Jiang H, Chait BT, Rout MP, Jensen TH, LaCava J. 2012. Improved methodology for the affinity isolation of human protein complexes expressed at near endogenous levels. Biotechniques 0: $1-6$.
LaCava J, Chandramouli N, Jiang H, Rout MP. 2013. Improved native isolation of endogenous protein A-tagged protein complexes. Biotechniques 54: 213-216.

LaCava J, Fernandez-Martinez J, Hakhverdyan Z, Rout MP. 2015a. Protein complex purification by affinity capture. Cold Spring Harb Protoc doi: 10.1101/pdb.top077545.

LaCava J, Fernandez-Martinez J, Rout MP. 2015b. Native elution of yeast protein complexes obtained by affinity capture. Cold Spring Harb Protoc doi: $10.1101 /$ pdb.prot087940.

Oeffinger M, Wei KE, Rogers R, DeGrasse JA, Chait BT, Aitchison JD, Rout MP. 2007. Comprehensive analysis of diverse ribonucleoprotein complexes. Nat Methods 4: 951-956. 


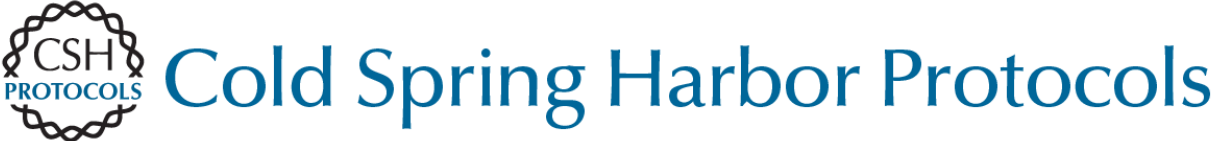

\section{Optimized Affinity Capture of Yeast Protein Complexes}

John LaCava, Javier Fernandez-Martinez, Zhanna Hakhverdyan and Michael P. Rout

Cold Spring Harb Protoc; doi: 10.1101/pdb.prot087932

\begin{tabular}{|c|c|}
\hline $\begin{array}{l}\text { Email Alerting } \\
\text { Service }\end{array}$ & Receive free email alerts when new articles cite this article - click here. \\
\hline $\begin{array}{r}\text { Subject } \\
\text { Categories }\end{array}$ & $\begin{array}{l}\text { Browse articles on similar topics from Cold Spring Harbor Protocols. } \\
\text { Characterization of Protein Complexes ( } 83 \text { articles) } \\
\text { Characterization of Proteins ( } 208 \text { articles) } \\
\text { Immunoaffinity Purification ( } 49 \text { articles) } \\
\text { Preparation of Cellular and Subcellular Extracts (104 articles) } \\
\text { Protein Identification and Analysis ( } 202 \text { articles) } \\
\text { Proteins and Proteomics, general (575 articles) } \\
\text { Yeast (288 articles) }\end{array}$ \\
\hline
\end{tabular}

